\title{
微波辅助 $\alpha$-重氮酯类化合物的快速合成
}

\author{
易享炎 ${ }^{a} \quad$ 张志朋 $^{a} \quad$ 黄 和 ${ }^{a} \quad$ Jonathan B. Baell $^{a}$ \\ 于 杨 $*$, 黄 菲 $*, a$ \\ ( ${ }^{a}$ 南京工业大学药学院 南京 211816) \\ ( ${ }^{b}$ 南京工业大学环境科学与工程学院 南京 211816)
}

\begin{abstract}
摘要 近年来, 微波辅助有机合成(Microwave-assisted organic synthesis, MAOS)作为新兴的绿色有机合成方法受到研究 者的广泛关注. 与传统加热方法相比，其反应时间更短，产物收率更高并具有良好的反应选择性. 许多人名反应中，譬 如 Diels-Alder 反应、Witting 反应、Heck 反应和 Mannich 反应，均可利用该技术实现反应的快速进行. 另外， $\alpha$-重氮酯 类化合物作为有机合成中一类重要的活性中间体，可以应用于 Wolff 重排反应以及 $\mathrm{C}-\mathrm{H}, \mathrm{N}-\mathrm{H}, \mathrm{Si}-\mathrm{H}$ 等一系列插入 反应. 虽然关于 $\alpha$-重氮酯的研究在 20 年前已经成为一个成熟的学科, 但在一些领域仍激发着研究者浓厚的研究兴趣. 基于此，借助微波技术实现了短时间内 $\alpha$-重氮酯类化合物的克级制备，分离收率最高可达 $90 \%$, 且具有良好的官能团 兼容性. 该方法操作简便，反应条件温和，原子利用效率高，符合绿色化学的要求，为 $\alpha$-重氮酯类化合物的合成及进一 步衍生化研究提供极大的便利.
\end{abstract}

关键词 微波辅助; $\alpha$-重氮酯; 绿色化学

\section{Microwave-Assisted Synthesis of $\alpha$-Diazoesters

\author{
Yi, Xiangyan ${ }^{a} \quad$ Zhang, Zhipeng $^{a} \quad{\text { Huang, } \mathrm{He}^{a} \quad \text { Baell, Jonathan B. }}^{a}$ \\ Yu, Yang*,b Huang, Fei*,a \\ ( ${ }^{a}$ College of Pharmaceutical Sciences, Nanjing Tech Univesity, Nanjing 211816) \\ ( ${ }^{b}$ School of Environmental and Engineering, Nanjing Tech University, Nanjing 211816)
}

\begin{abstract}
Shorter reaction times, higher product yields and enhanced selectivity are some of the outstanding advantages over conventional methods that the microwave-assisted organic synthesis possesses, which makes this methods develop into a significant mainstream both in industrial usages and academic researches. Microwave-assisted organic synthesis as a new protocol for green chemistry showcases the application of microwave heating in a number of areas of preparative chemistry as well as in the biosciences. Many name reactions can be performed by microwave-assisted, including the Diels-Alder reaction, the Witting reaction, the Heck reaction and the Mannich reaction. Meanwhile, $\alpha$-diazoester was already a mature science 20 years ago, some areas that continue to attract the most attention are preparation of diazoester substrates, such as the Wolff rearrangement, $\mathrm{C}-\mathrm{H}$ insertion, $\mathrm{N}-\mathrm{H}$ insertion, $\mathrm{Si}-\mathrm{H}$ insertion and so on. Therefore, it is necessary to develop a green and efficient method for the preparation of $\alpha$-diazoester. Herein a new method of microwave-assisted synthesis of $\alpha$-diazoesters compounds from 2-phenylacetates and tosyl azide in $30 \mathrm{~min}$ is described. The protocol provides a quick, efficient and green approach to various $\alpha$-diazoesters compounds with up to $90 \%$ isolated yields and a broad range of functional groups.
\end{abstract}

Keywords microwave-assisted; $\alpha$-diazoester; green chemistry

自 1991 年 “绿色化学” 的概念被提出以来, 有机合 成的绿色化一直备受关注. 在传统的有机合成中, 通常 需要维持较高的温度、较长的反应时间以及使用有毒且 生物降解性差的有机试剂作为溶剂, 这一过程无疑会给
能源和环境造成巨大的负担. 近年来, 传统的有机合成 方法正在被一些绿色、快速、高效的合成方法所取代，譬 如微波辅助有机合成、声化学合成技术、光催化、电催 化、酶催化以及非传统溶剂有机合成 ${ }^{[1]}$. 其中, 微波辅助

\footnotetext{
* Corresponding authors. E-mail: yuyang19880421@yeah.net; huangfei0208@yeah.net Received July 20, 2018; revised September 7, 2018; published September 26, 2018.

Project supported by the Jiangsu Synergetic Innovation Center for Advanced Bio-manufacture (Nos. XTE1850, XTC1810) and the Program for Innovative Research Team in Universities of Jiangsu Province (2015).

江苏省先进生物制造创新中心(Nos. XTE1850, XTC1810)和江苏高校优秀科技创新团队计划(2015)资助项目
} 
有机合成(MAOS)由于其加热速率快、操作方便、产率 高等特点被认为是一种极具应用前景的绿色合成方法 [2].

微波应用于有机合成的研究始于 1986 年, Gedye 等 ${ }^{[3]}$ 通过比较常规条件与微波辐射条件下进行酯化、水 解、氧化等反应, 发现在微波辐射下, 反应得到了不同 程度的加快. 微波加热的热量产生于物质内部是它区别 于其他加热方法的最主要的特点 ${ }^{[4]}$. 目前, 微波加快有 机合成反应的类型众多 ${ }^{[5]}$, 包括 Diels-Alder 反应、 Witting 反应、Claisen 重排反应、Mannich 反应、Heck 反应、烯烃加成、酯化反应、烷基化反应、脱保护反应、 成环反应、缩合反应、偶合反应、硝化反应、水解反应 等, 反应类型几乎涉及了有机合成反应的各个主要领 域, 基本上在传统加热条件下的能发生的有机反应都可 以在微波下进行.

$\alpha$-重氮酯类化合物是一种有机合成中应用极为广 泛的活性中间体 ${ }^{[6]}$, 此类化合物分解后失去 $\mathrm{N}_{2}$, 根据不 同的反应体系形成高度活泼的自由基卡宾或金属卡宾, 可以用于 $\mathrm{C}-\mathrm{H}, \mathrm{N}-\mathrm{H}, \mathrm{O}-\mathrm{H}, \mathrm{Si}-\mathrm{H}, \mathrm{B}-\mathrm{H}, \mathrm{C}-\mathrm{C}$ 或 $\mathrm{C}=\mathrm{C}$ 插入反应构筑相应的 $\mathrm{C}-\mathrm{C}, \mathrm{C}-\mathrm{N}, \mathrm{C}-\mathrm{O}, \mathrm{C}-\mathrm{Si}$,
$\mathrm{C}-\mathrm{B}$ 键等, 具有良好的化学反应活性及选择性 ${ }^{[7]}$ (Scheme 1).

传统借助重氮转移反应实现 $\alpha$-重氮酯类化合物的 合成, 通常需要持续加热 ${ }^{[8]}$ 或室温下搅拌过夜 ${ }^{[9]}$.

因此，我们希望以此作为研究的切入点，将微波技 术应用于 $\alpha$-重氮酯类化合物的合成，这种方法可以在短 时间内实现 $\alpha$-重氮酯类化合物的克级制备，且具有很好 的底物普适性，从而为 $\alpha$-重氮酯类化合物的制备提供一 种绿色、高效的合成路径(Scheme 2).

\section{1 结果与讨论}

为了篎选最佳反应条件, 以苯乙酸甲酯(1a) 和对甲 苯磺酰叠氮(2a)为原料, 1,8-二氮杂双环 [5.4.0]十一碳-7烯(DBU)为催化剂, 以乙腈为溶剂, 在微波反应器中反 应 $30 \mathrm{~min}$ 为模型反应条件，考察微波功率、温度、时间 及物料投料比对产率的影响(表 1).

实验结果表明微波功率对产率有一定的影响, 微波 功率为 $400 \mathrm{~W}$ 时产率最高, 加大微波功率产率反而下 降，这可能是由于功率过大，升温速率太快导致部分原

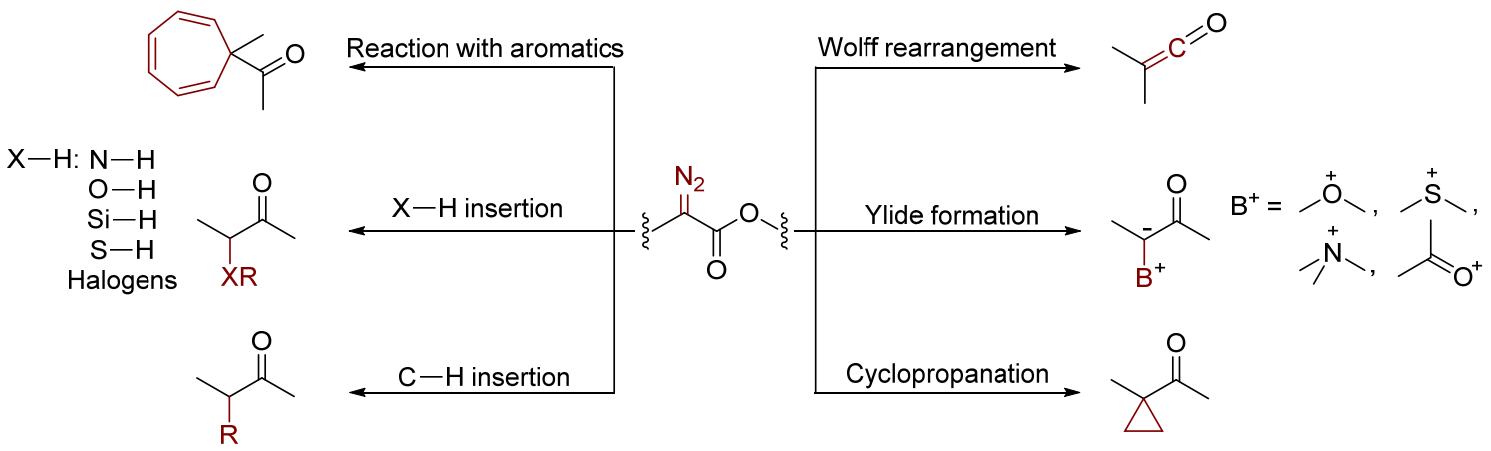

图式 $1 \alpha$-重氮酯的应用

Scheme 1 Application of $\alpha$-diazoesters

Pervious work
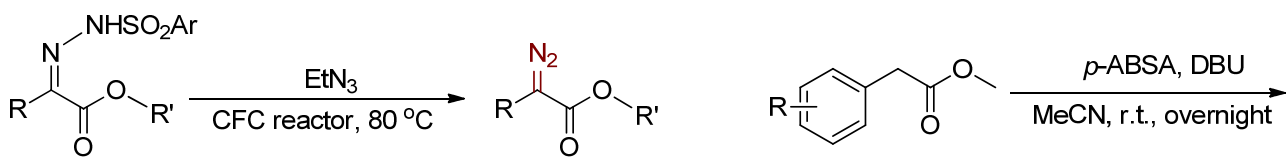<smiles>[R]=[R]1ccc(C(=N)C(=O)OC)cc1</smiles>

This work

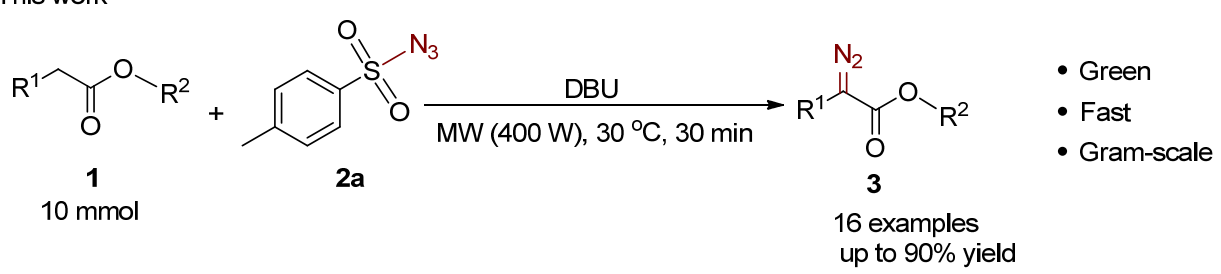

图式 $2 \alpha$-重氮酯的合成途径

Scheme 2 Approach to $\alpha$-diazoesters 
料发生分解变质; 在微波功率为 $400 \mathrm{~W}$ 的条件下, 又对 微波加热的温度进行考察, 实验结果表明, 反应温度过 低或过高都不利于反应的进行, $30{ }^{\circ} \mathrm{C}$ 为最佳反应温度; 对微波加热的时间进行考察, 发现反应时间为 $30 \mathrm{~min}$ 时，原料的产率即可达到 $90 \%$, 且随着反应时间的延长, 产率不再提高.

在此基础上，继续探究了不同物料比对反应产率的 影响. 发现当 $1 \mathbf{a} / 2 \mathrm{a}$ 物质的量之比为 $1: 1.1$ 时, 产率最 高. 至此, 该反应的最优条件为: $1 \mathrm{a}(0.5 \mathrm{mmol}), 2 \mathrm{a}(0.55$ mmol, 1.1 equiv.), DBU ( $0.75 \mathrm{mmol}, 1.5$ equiv.), $\mathrm{MeCN}$ (5 $\mathrm{mL})$, 微波功率 $400 \mathrm{~W}, 30{ }^{\circ} \mathrm{C}, 30 \mathrm{~min}$, 气相收率为 $95 \%$, 分离收率为 $90 \%$.

表 1 条件䇻选 ${ }^{a}$

Table 1 Conditions optimization

\begin{tabular}{ccccc}
\hline Entry & Power $/ \mathrm{W}$ & Temp. $/{ }^{\circ} \mathrm{C}$ & Time $/ \mathrm{min}$ & Yield $^{b} / \%$ \\
\hline 1 & 200 & 30 & 30 & 80 \\
2 & 400 & 30 & 30 & 90 \\
3 & 600 & 30 & 30 & 86 \\
4 & 800 & 30 & 30 & 82 \\
5 & 400 & 40 & 30 & 90 \\
6 & 400 & 60 & 30 & 88 \\
7 & 400 & 80 & 30 & 80 \\
8 & 400 & 30 & 10 & 75 \\
9 & 400 & 30 & 40 & 90 \\
10 & 400 & 30 & 60 & 90 \\
$11^{c}$ & 400 & 30 & 30 & $95(90)^{d}$ \\
$12^{e}$ & 400 & 30 & 30 & 88 \\
$13^{f}$ & 400 & 30 & 30 & 85 \\
\hline
\end{tabular}

${ }^{a}$ Conditions: 1a $(0.5 \mathrm{mmol}), 2 \mathrm{a}(0.5 \mathrm{mmol}), \mathrm{DBU}(0.7 \mathrm{mmol}), \mathrm{MeCN}(5 \mathrm{~mL})$, air. ${ }^{b} \mathrm{GC}$ yield with mesitylene as the internal standard. ${ }^{c} \mathbf{2 a}(0.55 \mathrm{mmol})$.

${ }^{d}$ Isolated yield given in parentheses. ${ }^{e} \mathbf{2 a}(0.65 \mathrm{mmol}) .{ }^{f} \mathbf{2 a}(1.0 \mathrm{mmol})$.

得到最优的条件后, 开始对该反应体系的底物适用 范围进行了考察(表 2). $\mathrm{R}^{1}$ 选自以下基团: 乙酰基、乙基、 苠基或卤素、三氟甲基、甲基、甲氧基、对甲苯磺酰基 取代的苯环; $\mathrm{R}^{2}$ 为甲基、乙基、烯丙基、异丁基和苄基. 首先探究了苯环上的电子效应和位阻效应对收率的影 响，发现含有吸电子基取代的 $3 \mathbf{b} \sim 3 \mathbf{f}$ 的收率在 80\% 86\%之间，供电子基取代的 3g，3h 收率可以达到 85\%; 不同位置取代 $3 \mathrm{c} \sim 3 \mathrm{e}$ 之间收率相差不明显, 均可以中 等至良好的收率得到目标产物, 电子效应和位阻效应对 目标产物收率影响不大. 其次, 对于不同类型的酯, 例 如乙酯 $(3 g \sim 3 i) 、$ 烯丙基酯 $(3 \mathbf{j}) 、$ 异丁酯 $(3 \mathbf{k}) 、$ 苠酯 $(3 \mathrm{l})$, 其 目标产物的收率也可以达到 $80 \%$ 以上, 均可实现克级规 模产物制备, 说明该反应体系具有较好的普适性. 值得 一提的是, 产物 $3 \mathrm{~m}$ 能够在一步反应中完成两个位点的 反应，在发生重氮转移反应的同时，对甲苯磺酰基会取 代苯环羟基上的氢，实现对差基的保护. 当原料 1 中不 含苯基, 即乙酰乙酸乙酯(1n)作为原料时, 反应也可以
很好地进行，以 $85 \%$ 的分离收率得到目标产物 $3 \mathrm{n}$. 当以 羰基 $\alpha$-位被乙基(10)或茮基(1p)取代的酯为原料时，在标 准条件下可实现乙酰基脱除进而得到目标产物 30 和 $\mathbf{3 p}$.

表 $2 \alpha$-重氮酯类化合物的底物拓展 ${ }^{a}$

Table 2 Substrates scope of $\alpha$-diazoesters compounds
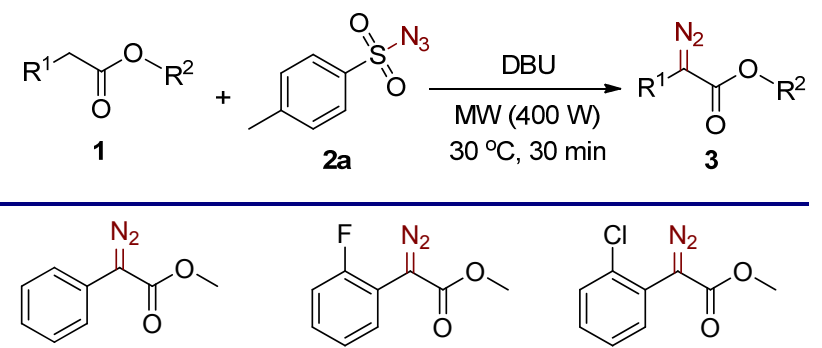

3a, $90 \%$

3b, $80 \%$

3c, $86 \%$<smiles>CCOC(=O)C(=N)c1ccc(C(F)(F)F)cc1</smiles><smiles>CCOC(=O)C(=N)c1ccc(C(=O)C(=N)c2ccc(OC)cc2)cc1</smiles><smiles>C=CCOC(=O)C(=N)c1ccccc1</smiles>

3j, $85 \%$

$3 \mathbf{k}, 80 \%$

3I, $80 \%$

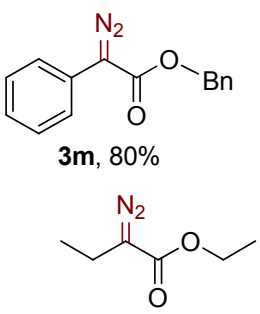

3o, $80 \%$<smiles>CCOC(=O)C(=N)C(C)=O</smiles>

$3 n, 85 \%$<smiles>CCOC(=O)C(=N)Br</smiles>

$3 p, 80 \%$
${ }^{a}$ The reaction condition: 1a $(10.0 \mathrm{mmol}), \mathbf{2 a}(11.0 \mathrm{mmol}, 1.1$ equiv.), DBU (15.0 mmol, 1.5 equiv.), $\mathrm{MeCN}(20 \mathrm{~mL})$, microwave $400 \mathrm{~W}, 30{ }^{\circ} \mathrm{C}, 30 \mathrm{~min}$ Isolated yields.

此外, 我们对产物 3a 做了进一步衍生化, 重氮酯 3a 作为卡宾插入前体，与对三氟甲基苯胺反应，实现了 卡宾插入 $\mathrm{N}-\mathrm{H}$ 键, 构筑了新的 $\mathrm{C}-\mathrm{N}$ 键 ${ }^{[10]}$, 得到了 2-((4-(三氟甲基)苯基)氨基)苯乙酸甲酯(5a). 同样使用 金属铑，可以实现产物 $3 \mathrm{~g}$ 与苯酚之间 $\mathrm{O}-\mathrm{H}$ 插入反 应 $^{[11]}$, 得到了 2-苯氧基 2-苯基乙酸乙酯 $\mathbf{5 b}$ (Scheme 3).

苯乙酸甲酯 $(1 \mathbf{a})$ 和对甲苯磺酰叠氮 $(2 \mathrm{a})$ 的重氮转移 反应可能的反应机理如 Scheme 4 所描述 ${ }^{[12]}$. 首先，化合 物 $1 \mathrm{a}$ 中的 $\alpha-\mathrm{H}$ 在碱的作用下脱去, 形成碳负离子 $\mathbf{A}$, 继 而碳负离子亲核进攻氮正离子，经历过渡态 $\mathbf{B}$ 后，发生 分子内氢质子迁移，脱去 $\mathrm{Tos}-\mathrm{NH}^{-}$后得到终产物 $3 \mathbf{a}$. 
<smiles>COC(=O)C(Nc1ccc(C(F)(F)F)cc1)c1ccccc1</smiles>

5a, $79 \%$

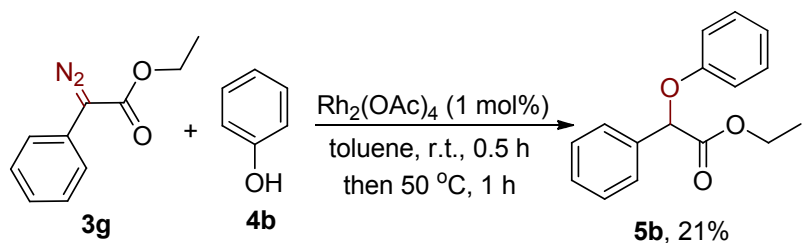

图式 $3 \mathrm{~N}-\mathrm{H}$ 和 $\mathrm{O}-\mathrm{H}$ 插入反应

Scheme $3 \mathrm{~N}-\mathrm{H}$ and $\mathrm{O}-\mathrm{H}$ insertion reaction

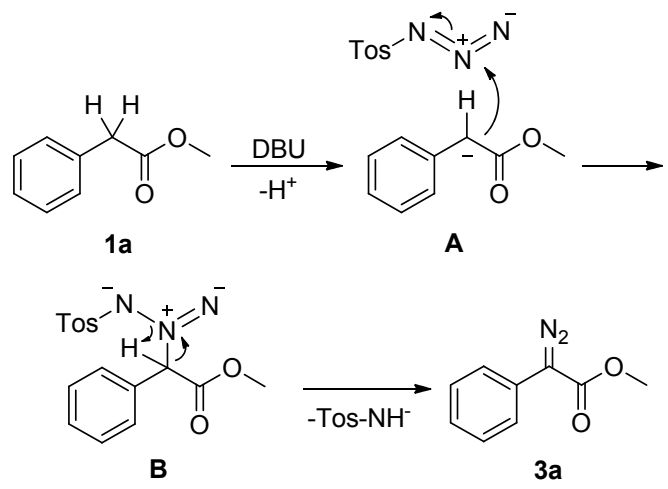

图式 4 可能的反应机理

Scheme 4 Plausible mechanism

\section{2 结论}

将微波技术应用于 $\alpha$-重氮酯类化合物的合成是一 种绿色有机合成策略. 通过对微波功率、温度、时间及 反应物料比的条件优化实现了 $\alpha$-重氮酯类化合物的高 效制备. 该方法不仅操作简单、绿色环保、官能团兼容 性良好, 而且有机反应速率相比传统的加热方法快数 倍, 能在短时间内进行克级规模产物制备, 为此类化合 物的合成提供了一种高效、便捷的方法. 同时, 对于含 有杂环、共轭结构更为复杂的 $\alpha$-重氮酯微波合成的研究 正在进行之中.

\section{3 实验部分}

\section{1 仪器与试剂}

选用南京信博仪器有限公司的 MC2-3A 常压微波 反应器. ${ }^{1} \mathrm{H}$ NMR 和 ${ }^{13} \mathrm{C}$ NMR 实验使用 Bruker DRX-400 核磁共振谱仪, 并以 $\mathrm{CDCl}_{3}$ 为溶剂, 以四甲基硅烷 (TMS)为内标. 气质分析使用 Agilent 7890/5975C 气质 联用仪. 薄层色谱(TLC)分析使用 Sigma-Aldrich silica gel $60 \mathrm{~F}_{200}$ 硅胶板, 柱层析使用 $200 \sim 300$ 目硅胶. 实验
中所使用溶剂均做无水处理，其他试剂未做进一步纯化 或处理.

\section{2 实验方法}

将 $100 \mathrm{~mL}$ 两口反应瓶置于 $0{ }^{\circ} \mathrm{C}$ 冰浴中, 加入酯类 化合物 $1(10.0 \mathrm{mmol})$ 和 $20 \mathrm{~mL}$ 乙腈, 搅拌均匀后加入对 甲苯磺酰叠氮 $(\mathbf{2 a}, 11.0 \mathrm{mmol})$, 缓慢加入 1,8 二二氮杂双 环 [5.4.0]十一碳-7-烯(DBU) (15.0 mmol), 在冰浴下搅拌 $5 \mathrm{~min}$, 放入微波反应器, 设定功率 $400 \mathrm{~W}$, 加热温度 $30{ }^{\circ} \mathrm{C}$, 加热时间 $30 \mathrm{~min}$, 开启排风扇. 反应结束后, 将 混合物冷却至室温, 加入 $\mathrm{NH}_{4} \mathrm{Cl}$ 溶液进行淬灭, 二氯甲 烷与水萃取, 收集有机相, 无水硫酸钠干燥, 过滤, 减 压下除去挥发组份, 然后用硅胶柱层析分离[洗脱液为 石油醚 $\left(60 \sim 90{ }^{\circ} \mathrm{C}\right)$ /乙酸乙酯, $V: V=100: 1$ ] 得到目标 产物 3.

2-重氮基-2-苯基乙酸甲酯(3a $)^{[13]}$ : 红色油状液体, $1.58 \mathrm{~g}$, 产率 $90 \% .{ }^{1} \mathrm{H}$ NMR $\left(400 \mathrm{MHz}, \mathrm{CDCl}_{3}\right) \delta: 7.49$ (dd, $J=8.5,1.1 \mathrm{~Hz}, 2 \mathrm{H}), 7.39$ (t, $J=7.9 \mathrm{~Hz}, 2 \mathrm{H}), 7.19(\mathrm{t}$, $J=7.4 \mathrm{~Hz}, 1 \mathrm{H}), 3.87$ (s, 3H); ${ }^{13} \mathrm{C} \mathrm{NMR}\left(100 \mathrm{MHz}, \mathrm{CDCl}_{3}\right)$ $\delta: 165.57,128.96,125.83,125.49,123.94,51.99$. 未在核 磁谱中监测到与重氮基相连碳原子的峰.

2-重氮基-2-(2-氟苯基)乙酸甲酯(3b $)^{[14]}$ : 黄色油状 液体, $1.55 \mathrm{~g}$, 产率 $80 \% .{ }^{1} \mathrm{H}$ NMR $\left(400 \mathrm{MHz}, \mathrm{CDCl}_{3}\right) \delta$ : $7.70(\mathrm{t}, J=8.7 \mathrm{~Hz}, 1 \mathrm{H}), 7.29 \sim 7.14(\mathrm{~m}, 2 \mathrm{H}), 7.13 \sim 7.001$ $(\mathrm{m}, 1 \mathrm{H}), 3.86(\mathrm{~s}, 3 \mathrm{H}) ;{ }^{13} \mathrm{C}$ NMR $\left(100 \mathrm{MHz}, \mathrm{CDCl}_{3}\right) \delta$ : 165.66, 158.41 (d, $J=247.4 \mathrm{~Hz}), 129.47(\mathrm{~d}, J=2.00 \mathrm{~Hz})$, $128.61(\mathrm{~d}, J=8.2 \mathrm{~Hz}), 124.67(\mathrm{~d}, J=3.5 \mathrm{~Hz}), 115.70$ (d, $J=21.3 \mathrm{~Hz}), 113.81(\mathrm{~d}, J=11.9 \mathrm{~Hz}), 52.20$. 未在核磁谱 中监测到与重氮基相连碳原子的峰.

2-重氮基-2-(2-氯苯基)乙酸甲酯(3c) ${ }^{[15]}$ : 黄色油状 液体, $1.81 \mathrm{~g}$, 产率 $86 \% .{ }^{1} \mathrm{H}$ NMR $\left(400 \mathrm{MHz}, \mathrm{CDCl}_{3} \delta\right.$ : $7.55(\mathrm{dd}, J=7.7,1.6 \mathrm{~Hz}, 1 \mathrm{H}), 7.43(\mathrm{dd}, J=7.8,1.4 \mathrm{~Hz}$, $1 \mathrm{H}), 7.37 \sim 7.26(\mathrm{~m}, 2 \mathrm{H}), 3.85(\mathrm{~s}, 3 \mathrm{H}) ;{ }^{13} \mathrm{C}$ NMR $(100$ $\left.\mathrm{MHz}, \mathrm{CDCl}_{3}\right) \delta: 166.06,133.84,132.39,130.17,129.72$, $127.25,123.98,52.38$.

2-重氮基-2-(3-氯苯基)乙酸甲酯 $(3 \mathbf{d})^{[15]}$ : 黄色固体, $1.75 \mathrm{~g}$, 产率 $83 \%$. m.p. $50 \sim 52{ }^{\circ} \mathrm{C} ;{ }^{1} \mathrm{H}$ NMR $(400 \mathrm{MHz}$, $\left.\mathrm{CDCl}_{3}\right) \delta: 7.53(\mathrm{~s}, 1 \mathrm{H}), 7.36 \sim 7.27(\mathrm{~m}, 2 \mathrm{H}), 7.14(\mathrm{dt}, J=$ 7.1, $1.9 \mathrm{~Hz}, 1 \mathrm{H}), 3.87$ (s, 3H); ${ }^{13} \mathrm{C}$ NMR $(100 \mathrm{MHz}$, $\left.\mathrm{CDCl}_{3}\right) \delta: 165.13,135.13,130.15,127.81,125.87,123.76$, $121.67,63.33,52.25$.

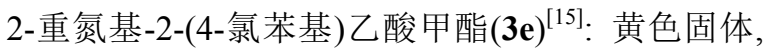
$1.68 \mathrm{~g}$, 产率 80\%. m.p. $50 \sim 53{ }^{\circ} \mathrm{C} ;{ }^{1} \mathrm{H}$ NMR $(400 \mathrm{MHz}$, $\left.\mathrm{CDCl}_{3}\right) \delta: 7.42(\mathrm{~d}, J=8.8 \mathrm{~Hz}, 2 \mathrm{H}), 7.35(\mathrm{~d}, J=8.9 \mathrm{~Hz}$, 2H), $3.87(\mathrm{~s}, 3 \mathrm{H}) ;{ }^{13} \mathrm{C}$ NMR $\left(100 \mathrm{MHz}, \mathrm{CDCl}_{3}\right) \delta: 165.42$, $131.60,129.23,125.17,124.24,52.24$. 未在核磁谱中监 
测到与重氮基相连碳原子的峰.

2-重氮基-2-(4-三氟甲基苯基)乙酸乙酯(3f) ${ }^{[16]}$ : 黄 色固体, $2.06 \mathrm{~g}$, 产率 $80 \%$. m.p. 59 60 ${ }^{\circ} \mathrm{C} ;{ }^{1} \mathrm{H} \mathrm{NMR}$ $\left(400 \mathrm{MHz}, \mathrm{CDCl}_{3}\right) \delta: 7.61(\mathrm{~s}, 4 \mathrm{H}), 4.35$ (q, $J=7.1 \mathrm{~Hz}$, $2 \mathrm{H}), 1.35$ (t, $J=7.1 \mathrm{~Hz}, 3 \mathrm{H}) ;{ }^{13} \mathrm{C} \mathrm{NMR}\left(100 \mathrm{MHz}, \mathrm{CDCl}_{3}\right)$ $\delta: 164.44,130.25,127.59$ (d, $J=32.8 \mathrm{~Hz}), 125.94$ (q, $J=$ $3.8 \mathrm{~Hz}), 124.08$ (q, $J=271.6 \mathrm{~Hz}), 123.41,61.33,14.47$.

2-重氮基-2-(4-甲基苯基)乙酸乙酯 $(\mathbf{3 g})^{[17]}$ : 红色油 状液体, $1.73 \mathrm{~g}$, 产率 $85 \%$. ${ }^{1} \mathrm{H}$ NMR $\left(400 \mathrm{MHz}, \mathrm{CDCl}_{3}\right) \delta$ : 7.37 (d, $J=8.3 \mathrm{~Hz}, 2 \mathrm{H}), 7.20$ (d, $J=8.1 \mathrm{~Hz}, 2 \mathrm{H}), 4.33$ (q, $J=7.1 \mathrm{~Hz}, 2 \mathrm{H}), 2.34(\mathrm{~s}, 3 \mathrm{H}), 1.34(\mathrm{t}, J=7.1 \mathrm{~Hz}, 3 \mathrm{H}) ;{ }^{13} \mathrm{C}$ NMR $\left(100 \mathrm{MHz}, \mathrm{CDCl}_{3}\right) \delta: 165.62,135.76,129.78$, $124.23,122.39,61.04,21.12,14.64$.

2-重氮基-2-(4-甲氧基苯基)乙酸甲酯(3h) ${ }^{[15]}$ : 红色 固体, $1.75 \mathrm{~g}$, 产率 85\%. m.p. $45 \sim 48{ }^{\circ} \mathrm{C} ;{ }^{1} \mathrm{H}$ NMR (400 $\left.\mathrm{MHz}, \mathrm{CDCl}_{3}\right) \delta: 7.38(\mathrm{~d}, J=9.0 \mathrm{~Hz}, 2 \mathrm{H}), 6.94(\mathrm{~d}, J=9.0$ $\mathrm{Hz}, 2 \mathrm{H}), 3.85$ (s, 3H), 3.80 (s, 3H); ${ }^{13} \mathrm{C} \mathrm{NMR} \mathrm{(100} \mathrm{MHz,}$ $\left.\mathrm{CDCl}_{3}\right) \delta: 166.26,158.17,126.05,116.94,114.69,55.43$, 52.05. 未在核磁谱中监测到与重氮基相连碳原子的峰.

2-重氮基-2-(4-(对甲苯磺酰氧基)苯基)乙酸甲酯 $(3 \mathrm{i})^{[18]}$ : 橙色固体, $2.60 \mathrm{~g}$, 产率 $75 \%$. m.p. $101 \sim 102{ }^{\circ} \mathrm{C}$; ${ }^{1} \mathrm{H}$ NMR (400 MHz, $\left.\mathrm{CDCl}_{3}\right) \delta: 7.69$ (d, $\left.J=8.3 \mathrm{~Hz}, 2 \mathrm{H}\right)$, 7.39 (d, $J=8.9 \mathrm{~Hz}, 2 \mathrm{H}), 7.30$ (d, $J=8.1 \mathrm{~Hz}, 2 \mathrm{H}), 6.98$ (d, $J=8.9 \mathrm{~Hz}, 2 \mathrm{H}), 3.84$ (s, 3H), 2.44 (s, 3H); ${ }^{13} \mathrm{C}$ NMR (100 $\left.\mathrm{MHz}, \mathrm{CDCl}_{3}\right) \delta: 165.32,147.39,145.60,132.28,129.92$, $128.65,124.87,123.10,52.23,21.85$. 未在核磁谱中监测 到与重氮基相连碳原子的峰.

2-重氮基-2-苯基乙酸乙酯 $(\mathbf{3} \mathbf{j})^{[19]}$ : 红色油状液体, $1.62 \mathrm{~g}$, 产率 $85 \%$. ${ }^{1} \mathrm{H}$ NMR (400 $\left.\mathrm{MHz}, \mathrm{CDCl}_{3}\right) \delta: 7.50$ (d, $J=7.5 \mathrm{~Hz}, 2 \mathrm{H}), 7.39$ (t, $J=7.9 \mathrm{~Hz}, 2 \mathrm{H}), 7.19$ (t, $J=7.4$ $\mathrm{Hz}, 1 \mathrm{H}), 4.34$ (q, $J=7.1 \mathrm{~Hz}, 2 \mathrm{H}), 1.35$ (t, $J=7.1 \mathrm{~Hz}, 3 \mathrm{H})$; ${ }^{13} \mathrm{C}$ NMR $\left(100 \mathrm{MHz}, \mathrm{CDCl}_{3}\right) \delta: 165.25,128.97,125.79$, $125.70,124.00,61.03,14.56$. 未在核磁谱中监测到与重 氮基相连碳原子的峰.

2-重氮基-2-苯基乙酸异丁酯(3k) ${ }^{[200]}$ : 黄色油状液 体, $1.73 \mathrm{~g}$, 产率 $80 \% .{ }^{1} \mathrm{H} \mathrm{NMR}\left(400 \mathrm{MHz}, \mathrm{CDCl}_{3}\right) \delta: 7.49$ (d, $J=7.5 \mathrm{~Hz}, 2 \mathrm{H}), 7.39$ (t, $J=7.9 \mathrm{~Hz}, 2 \mathrm{H}), 7.18$ (t, $J=7.4$ $\mathrm{Hz}, 1 \mathrm{H}), 4.07$ (d, $J=6.6 \mathrm{~Hz}, 2 \mathrm{H}), 2.02(\mathrm{dt}, J=13.4,6.7$ $\mathrm{Hz}, 1 \mathrm{H}), 0.98$ (d, $J=6.7 \mathrm{~Hz}, 6 \mathrm{H}) ;{ }^{13} \mathrm{C} \mathrm{NMR}(100 \mathrm{MHz}$, $\left.\mathrm{CDCl}_{3}\right) \delta: 165.37,129.05,125.88,125.75,124.06,71.06$, 28.04, 19.18 .

2-重氮基-2-苯基乙酸烯丙酯(31) ${ }^{[20]}$ : 红色油状液体, $1.62 \mathrm{~g}$, 产率 $80 \% .{ }^{1} \mathrm{H}$ NMR $\left(400 \mathrm{MHz}, \mathrm{CDCl}_{3}\right) \delta: 7.51(\mathrm{~d}$, $J=8.5 \mathrm{~Hz}, 2 \mathrm{H}), 7.40(\mathrm{t}, J=7.9 \mathrm{~Hz}, 2 \mathrm{H}), 7.20$ (t, $J=7.4$ $\mathrm{Hz}, 1 \mathrm{H}), 6.00$ (ddd, $J=22.8,10.8,5.6 \mathrm{~Hz}, 1 \mathrm{H}), 5.34$ (dd,
$J=35.3,13.8 \mathrm{~Hz}, 2 \mathrm{H}), 4.79(\mathrm{~d}, J=5.6 \mathrm{~Hz}, 2 \mathrm{H}) ;{ }^{13} \mathrm{C} \mathrm{NMR}$ $\left(100 \mathrm{MHz}, \mathrm{CDCl}_{3}\right) \delta: 164.80,132.15,128.96,125.86$, 125.46, 123.97, 118.36, 65.44.

2-重氮基-2-苯基乙酸苄酯 $(\mathbf{3 m})^{[21]}$ : 黄色固体, 2.02 g, 产率 80\%. m.p. 53 55 ${ }^{\circ} \mathrm{C} ;{ }^{1} \mathrm{H}$ NMR $(400 \mathrm{MHz}$, $\left.\mathrm{CDCl}_{3}\right) \delta: 7.51(\mathrm{~d}, J=7.5 \mathrm{~Hz}, 2 \mathrm{H}), 7.44 \sim 7.33(\mathrm{~m}, 7 \mathrm{H})$, $7.200(\mathrm{t}, J=7.4 \mathrm{~Hz}, 1 \mathrm{H}), 5.33(\mathrm{~s}, 2 \mathrm{H}) ;{ }^{13} \mathrm{C}$ NMR $(100$ $\left.\mathrm{MHz}, \mathrm{CDCl}_{3}\right) \delta: 165.11,135.98,129.06,128.74,128.45$, $128.27,125.99,125.51,124.10,66.59$. 未在核磁谱中监 测到与重氮基相连碳原子的峰.

2-重氮基-乙酰乙酸乙酯 $(3 n){ }^{[22]}$ : 黄色油状液体, $1.33 \mathrm{~g}$, 产率 $85 \% .{ }^{1} \mathrm{H}$ NMR $\left(400 \mathrm{MHz}, \mathrm{CDCl}_{3}\right) \delta: 4.29$ (q, $J=7.1 \mathrm{~Hz}, 2 \mathrm{H}), 2.46(\mathrm{~s}, 3 \mathrm{H}), 1.32(\mathrm{t}, J=7.1 \mathrm{~Hz}, 3 \mathrm{H}) ;{ }^{13} \mathrm{C}$ NMR (100 MHz, $\left.\mathrm{CDCl}_{3}\right) \delta: 190.38,161.55,61.56,28.37$, 14.45 .

2-重氮基丁酸乙酯 $(30)^{[23]}$ : 黄色油状液体, $1.14 \mathrm{~g}$, 产率 $80 \% .{ }^{1} \mathrm{H}$ NMR (400 MHz, $\mathrm{CDCl}_{3}$ ) $\delta: 4.18$ (q, $J=7.1$ $\mathrm{Hz}, 2 \mathrm{H}), 2.31$ (q, $J=7.5 \mathrm{~Hz}, 2 \mathrm{H}), 1.23$ (t, $J=7.1 \mathrm{~Hz}, 3 \mathrm{H})$, 1.09 (t, $J=7.5 \mathrm{~Hz}, 3 \mathrm{H}) ;{ }^{13} \mathrm{C}$ NMR $\left(100 \mathrm{MHz}, \mathrm{CDCl}_{3}\right) \delta$ : 167.59, 60.70, 16.61, 14.55, 11.96 .

2 -重氮基-3-苯基丙酸乙酯 $(3 p)^{[24]}$ : 黄色油状液体, $1.63 \mathrm{~g}$, 产率 $80 \% .{ }^{1} \mathrm{H}$ NMR $\left(400 \mathrm{MHz}, \mathrm{CDCl}_{3}\right) \delta: 7.35 \sim$ 7.29 (m, 2H), 7.25 (d, $J=7.5 \mathrm{~Hz}, 3 \mathrm{H}), 4.25$ (q, $J=7.1 \mathrm{~Hz}$, $2 \mathrm{H}), 3.63(\mathrm{~s}, 2 \mathrm{H}), 1.28(\mathrm{t}, J=7.1 \mathrm{~Hz}, 3 \mathrm{H}) ;{ }^{13} \mathrm{C} \mathrm{NMR}(100$ $\left.\mathrm{MHz}, \mathrm{CDCl}_{3}\right) \delta: 166.97,137.23,128.66,128.23,126.97$, $60.79,29.17,14.17$. 未在核磁谱中监测到与重氮基相连 碳原子的峰.

2- 苯基-2-((4-(三氟甲基) 苯基)氨基)乙酸甲酯 (5a) ${ }^{[25]}$ : 白色固体, $122 \mathrm{mg}$, 产率 79\%. m.p. 88 89 ${ }^{\circ} \mathrm{C}$; ${ }^{1} \mathrm{H}$ NMR (400 MHz, $\left.\mathrm{CDCl}_{3}\right) \delta: 7.48(\mathrm{~d}, J=6.7 \mathrm{~Hz}, 2 \mathrm{H})$, $7.43 \sim 7.28(\mathrm{~m}, 5 \mathrm{H}), 6.56(\mathrm{~d}, J=8.5 \mathrm{~Hz}, 2 \mathrm{H}), 5.38(\mathrm{~s}, 1 \mathrm{H})$, $5.11(\mathrm{~s}, 1 \mathrm{H}), 3.76(\mathrm{~s}, 3 \mathrm{H}) ;{ }^{13} \mathrm{C} \mathrm{NMR}\left(100 \mathrm{MHz}, \mathrm{CDCl}_{3}\right) \delta$ : $171.87,148.36,136.88,129.17,128.73,127.27,126.72$ (q, $J=3.7 \mathrm{~Hz}), 124.92(\mathrm{q}, J=270.3 \mathrm{~Hz}), 112.74,60.25,53.17$.

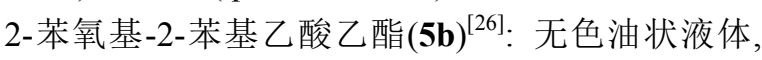
$25 \mathrm{mg}$, 产率 $21 \%$. ${ }^{1} \mathrm{H}$ NMR (400 $\left.\mathrm{MHz}, \mathrm{CDCl}_{3}\right) \delta: 7.52$ $(\mathrm{dd}, J=7.8,1.4 \mathrm{~Hz}, 2 \mathrm{H}), 7.35 \sim 7.26(\mathrm{~m}, 3 \mathrm{H}), 7.19$ (dd, $J=8.7,7.5 \mathrm{~Hz}, 2 \mathrm{H}), 6.99 \sim 6.81(\mathrm{~m}, 3 \mathrm{H}), 5.55(\mathrm{~s}, 1 \mathrm{H})$, 4.12 (dd, $J=14.0,7.1 \mathrm{~Hz}, 2 \mathrm{H}), 1.13$ (t, $J=7.1 \mathrm{~Hz}, 3 \mathrm{H})$; ${ }^{13} \mathrm{C}$ NMR (100 MHz, $\left.\mathrm{CDCl}_{3}\right) \delta: 170.06,157.40,135.59$, $129.65,129.02,128.86,127.16,121.87,115.55,78.71$, $61.74,14.13$.

辅助材料(Supporting Information) 化合物 $\mathbf{3 a} \sim \mathbf{3 p}$, $\mathbf{5 a}, \mathbf{5 b}$ 的 ${ }^{1} \mathrm{H} \mathrm{NMR}$ 和 ${ }^{13} \mathrm{C} \mathrm{NMR}$ 谱图. 这些材料可以免费 
从本刊网站(http://sioc-journal. cn/)上下载.

\section{References}

[1] (a) Feng, R.; Li, H. M. Sonochemistry and Its Applications, Anhui Science and Technology Press, Hefei, 1992 (in Chinese). (冯若, 李化茂, 声化学及其应用, 安徽科学技术出版社, 合肥, 1992.)

(b) Romero, N. A.; Nicewicz, D, A. Chem. Rev. 2016, 116, 10075.

(c) Shaw, M. H.; Twilton, J.; Macmillan, D. W. J. Org. Chem. 2016, 81, 6898 .

(d) Bockris, J. O.; Minevski, Z. S. Electrochim. Acta 1994, 39, 1471.

(e) Ming, Y.; Yu, K.; Baran, P. S. Chem. Rev. 2017, 117, 13230.

(f) Frank, R. A.; Leeper, F. J.; Luisi, B. F. Cell. Mol. Life Sci. 2007, $64,892$.

[2] (a) Kappe, C. O. Angew. Chem., Int. Ed. 2004, 43, 6250.

(b) Caddick, S. Tetrahedron Lett. 1995, 51, 10403.

(c) Pelle, L.; Jason, T.; Bernard, W.; Jacob, W. Tetrahedron 2001, 57, 9225.

(d) Enquist, P. A.; Nilsson, P.; Larhed, M. Org. Lett. 2003, 5, 4875.

(e) Sandoval, W.; Pham, V. J. Drug Discovery Today 2008, 13, 1075 .

(f) Appukkuttan, P.; Erik, V. D. E. Eur. J. Org. Chem. 2008, 7, 1133.

(g) Schmink, J. R.; Kormos, C. M.; Devine, W. G.; Leadbeater, N. E. Org. Process Res. Dev. 2010, 14, 205.

(h) Rodríguez, A. M.; Prieto, P.; De La Hoz, A.; Ángel DíazOrtiz; Martín, D. R.; García, J. I. ChemistryOpen 2015, 4, 308.

(i) Frecentese, F.; Saccone, I.; Caliendo, G.; Corvino, A.; Fiorino, F.; Magli, E.; Perissutti, E.; Severino, B.; Santagada, V. Med. Chem. 2016, 12,720

(j) Lin, W.; Zheng, Y. X.; Xun, Z.; Huang, Z. B.; Shi, D. Q. ACS Comb. Sci. 2017, 19, 708.

(k) Kumar, A.; Jad, Y. E.; Collins, J. M.; Albericio, F.; De la Torre, B. G. ACS Sustainable Chem. Eng. 2018, 6, 8034.

(1) Rieger, E.; Gleede, T.; Manhart, A.; Lamla, M.; Wurm, F. R. ACS Macro Lett. 2018, 7, 598.

(m) Verrier, C.; Carret, S.; Poisson, J. F. ACS Sustainable Chem. Eng. 2018, 6, 8563.

(n) Lin, J.; Wang, Y. L.; Zhang, J. H.; Yang, J. S.; Mou, H. C.; Lin, J.; Yan, S. J. ACS Omega 2018, 3, 4534.

[3] Gedye, R.; Smith, F.; Westaway, K. Tetrahedron Lett. 1986, 27, 279.

[4] Zhao, K. Y.; Xu, F. Y. The Principle and Technology of Microwave, Higher Education Press, Beijing, 2006 (in Chinese).

(赵克玉, 许福永, 微波原理与技术, 高等教育出版社, 北京, 2006.)

[5] Fan, X.; You, X.; Tan, G.; Yu, X.; Jiao, T. Prog. Chem. 1998, 10, 285 (in Chinese).

(樊兴君, 尤进茂, 谭干祖, 俞贤达, 焦天权, 化学进展, 1998, 10, 285.)

[6] (a) Ford, A.; Miel, H.; Ring, A.; Slattery, C. N.; Maguire, A. R.; Mckervey, M. A. Chem. Rev. 2015, 115, 9981.

(b) Orihara, K.; Kawagishi, F.; Yokoshima, S.; Fukuyama, T. Synlett 2018, 29, 769 .

(c) Deepa, N.; Prashant, P.; Savita, K.; Irishi, N. N. N. Tetrahedron Lett. 2016, 57, 3146.

(d) Rajasekaran, T.; Sridhar, B.; Reddy, B. V. S. Tetrahedron 2016, $72,2102$.

[7] (a) Ovalles, S. R.; Hansen, J. H.; Davies, H, M. Org. Lett. 2011, 13,
4284.

(b) Lebel, H.; Marcoux, J. F.; Molinaro, C.; Charette, A, B. Chem. Rev. 2003, 4, 977 .

(c) Nicolas, I.; Maux, P. L.; Simonneaux, G. Coord. Chem. Rev. 2008, 252, 727.

(e) Chen, D. Y.; Pouwer, R. H.; Richard, J. A. Chem. Soc. Rev. 2012, 41, 4631.

(f) Reisman, S. E.; Nani, R. R.; Levin, S. Synlett 2011, 2437.

(g) Davies, H. M.; Morton, D. Chem. Soc. Rev. 2011, 40, 1857.

(h) Xia, Y.; Liu, Z.; Liu, Z.; Ge, R.; Ye, F.; Hossain, M.; Zhang, Y.; Wang, J. J. Am. Chem. Soc. 2014, 136, 3031.

(i) Larsen, S. D.; Barf, T.; Liljebris, C.; May, P. D.; Ogg, D.; O'Sullivan, T. J.; Palazuk, B. J.; Schostarez, H. J.; Stevens, C. F.; Bleasdale, E. J. J. Med. Chem. 2002, 45, 598.

(j) Lepage, R. J.; White, J. M.; Coster, M. J. Chem. Commun. 2017, 53,4219 .

(k) Huang, B.; Shen, Y.; Mao, Z.; Liu, Y.; Cui, S. Org. Lett. 2016. 18,4888 .

(1) Popp, B. V.; Ball, Z. T. J. Am. Chem. Soc. 2010, 132, 6660.

[8] Bartrum, H. E.; Blakemore, D. C.; Moody, C. J.; Hayes, C. J. Chem.-Eur. J. 2011, 17, 9586.

[9] Keipour, H.; Jalba, A.; Delage-Laurin, L.; Ollevier, T. J. Org. Chem. 2017, 82, 3000.

[10] Nicolle, S. M.; Hayes, C. J.; Moody, C. J. Chemistry 2015, 21, 4576.

[11] Hazeldine, S. T.; Polin, L.; Kushner, J.; White, K.; Corbett, T. H.; Horwitz, J. P. Bioorg. Med. Chem. 2005, 13, 3910.

[12] (a) Danheiser, R. L.; Miller, R. F.; Brisbois, R. G.; Park, S. Z. J. Org. Chem. 1990, 55, 1959.

(b) Abad, A.; Agulló, C.; Cuñat, A. C.; de Alfonso Marzal, I.; Navarro, I.; Gris, A. Tetrahedron 2006, 62, 3266.

(c) Arthuis, M.; Beaud, R.; Gandon, V.; Roulland, E. Angew. Chem., Int. Ed. 2012, 51, 10510.

(d) Shibuya, G. M.; Enquist, J. A.; Stoltz, B. M. Org. Lett. 2013, 15, 3480 .

[13] Starmans, W. A. J.; Thijs, L.; Zwanenburg, B. Tetrahedron 1998, 54,629 .

[14] Maier, T. C.; Fu, G. C. J. Am. Chem. Soc. 2006, 128, 4594.

[15] Davies, H. M. L.; Hansen, T.; Churchill, M. R. J. Am. Chem. Soc. 2000, 122, 3063.

[16] Ye, F.; Wang, C.; Zhang, Y.; Wang, J. Angew. Chem., Int. Ed. 2014 53,11625 .

[17] Chen, G.; Song, J.; Yu, Y.; Luo, X.; Li, C.; Huang, X. Chem. Sci. 2016, 7, 1786.

[18] Jason, T.; Richard, T.; Graham K. M. J. Am. Chem. Soc. 2013, 135, 16312.

[19] Bachmann, S.; Fielenbach, D.; Jørgensen, K. A. Org. Biomol. Chem. 2004, 2, 3044.

[20] Luo, X.; Chen, G.; He, L.; Huang, X. J. Org. Chem. 2016, 81, 2943.

[21] Tayama, E.; Yanaki, T.; Iwamoto, H.; Hasegawa, E. Eur. J. Org. Chem. 2010, 2010, 6719 .

[22] Davies, J. R.; Kane, P. D.; Moody, C. J. Tetrahedron 2004, 60, 3967.

[23] Li, W.; Liu, X.; Hao, X.; Hu, X.; Chu, Y.; Cao, W.; Qin, S.; Hu, C.; Lin, L.; Feng, X. J. Am. Chem. Soc. 2011, 133, 15268.

[24] Taber, D. F.; Sheth, R. B.; Joshi, P. V. J. Org. Chem. 2005, 70, 2851.

[25] Wang, Y.; Zhu, Y.; Chen, Z.; Mi, A.; Hu, W.; Doyle, M. P. Org. Lett. 2003, 5, 3923.

[26] Zeineddine, A.; Rekhroukh, F.; Sosa Carrizo, E. D.; Mallet-Ladeira, S.; Miqueu, K.; Amgoune, A.; Bourissou, D. Angew. Chem., Int. Ed. 20018, 57, 1306 